\title{
Pharmacological quiescence for radiation-induced intestinal injury
}

A new study has shown that transiently inducing intestinal stem cell (ISC) quiescence with an inhibitor of cell cycle progression can protect against lethal radiation-induced intestinal injury in mice.

Radiation-induced gastrointestinal damage is a limiting factor in radiotherapy but no approved agent to treat this tissue injury currently exists. Previous work has shown that high-dose radiation leads to DNA double-strand breaks, activating the G1-S transition checkpoint as well as a rapid loss of ISCs via p53-dependent mechanisms. "We have been searching for ways and agents for intestinal protection that are likely to modulate the p53 pathway and DNA damage response," explains author Jian Yu. In vitro studies have shown that inhibiting cyclin-dependent kinases
(CDK) - critical proteins in the G1-S phase transition - suppresses radiosensitivity but these findings have not been well explored in vivo.

Now, using mouse models of abdominal and total body radiation and an FDA-approved inhibitor of CDK4 and CDK6 (CDKi), the investigators showed that mouse survival, crypt survival and the preservation of intestinal structure were all improved in mice treated with CDKi prior to radiation exposure compared with vehicle. Analysis of intestinal proliferation and apoptosis kinetics showed that CDKi treatment before radiation exposure blocked crypt proliferation and apoptosis but increased regeneration. CDKi treatment also enhanced survival of LRG5 ${ }^{+}$ISCs, which were shown to be primarily responsible for
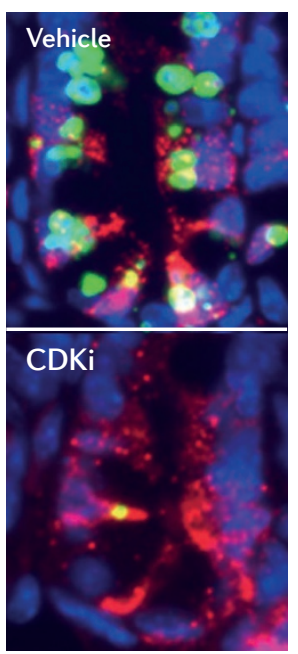

CDKi treatment blocks radiation-induced apoptosis (green) in LRG5 $5^{+}$(red) intestinal stem cells. Nuclei are blue. Courtesy of J. Yu. crypt regeneration using lineage tracing experiments.

The researchers found that $\mathrm{CDKi}$ treatment induced transient ISC quiescence, blocked p53-dependent ISC apoptosis and improved genome stability by influencing DNA repair mechanisms. All of these processes improved ISC quality, quantity and survival.

The team are now interested in demonstrating that this therapeutic approach selectively protects ISCs but not p53-deficient cancer cells, as well as identifying potential inhibitor combinations to further improve intestinal radioprotection. "We hope to be able to take our findings to the clinic to ... reduce radiation-induced intestinal injury and long-term complications," concludes Yu. "It would also be interesting to see if this approach can be extended to protect other radiosensitve or chemosensitive tissues."

Iain Dickson

ORIGINAL ARTICLE Wei, L. et al. Inhibition of CDK $4 / 6$ protects against radiation-induced intestinal injury in mice. J. Clin. Invest. http://dx.doi.org/10.1172/JCl88410 (2016) 